\title{
MODELO DE SISTEMA DE REFRIGERACIÓN DE EDIFICIOS ORIENTADO AL CONTROL Y GESTIÓN DE LA EFICIENCIA ENERGÉTICA
}

\author{
J.G. Ordonez ${ }^{1}$ \\ jgordonez@us.es \\ Luis Perez-Lombard ${ }^{2}$ \\ lpl@us.es
}

\author{
J.M. Nadales ${ }^{1}$ \\ nadales@us.es \\ Juan F. Coronel ${ }^{2}$ \\ jfc@us.es
}

\author{
D. Limon $^{1}$ \\ dlm@us.es
}

\author{
A. Cardona ${ }^{1}$ \\ acardona@us.es
}

1 Depto. Ingeniería de Sistemas y Automática. Esc. Técnica Superior de Ingeniería. Universidad de Sevilla.

${ }^{2}$ Depto. Ingeniería Energética. Esc. Técnica Superior de Ingeniería. Universidad de Sevilla.

\section{Resumen}

La climatización en edificios es una de las aplicaciones más importantes de los sistemas de refrigeración y su uso supone hasta un $20 \%$ de la energía consumida en países desarrollados. Actualmente, los ingenieros de control dedican grandes esfuerzos a mejorar la eficiencia energética de estos sistemas. Uno de los mayores obstáculos en esta línea de investigación es la obtención de buenos modelos, debido a la complejidad de las máquinas de refrigeración comerciales. En este artículo se presenta un modelo para un caso realista de estudio donde se incorporan máquinas reales para el estudio de técnicas de control para la mejora de la eficiencia. Además, se expone un ejemplo donde se aplica control predictivo basado en modelo con seguimiento de temperaturas.

Palabras clave: climatización, refrigeración, modelo económico, eficiencia energética, identificación basada en datos, control predictivo

\section{INTRODUCCIÓN}

Los sistemas de refrigeración tienen la misión de mantener la temperatura de un volumen de control por debajo de la temperatura de sus alrededores. Eso conlleva la transferencia de calor desde un espacio frío a otro caliente, en contra del gradiente térmico, y para ello es necesario realizar un ciclo de refrigeración. Una de las aplicaciones más importantes de los sistemas de refrigeración, y la que se trata en este artículo, es la de climatización.

En estudios actuales, se estima que en los países desarrollados, hasta un $40 \%$ del consumo de en- ergía se produce en edificios. Además, aproximadamente $50 \%$ de ese consumo se realiza por sistemas de climatización de cualquier tipo [11]. Esto evidencia la necesidad de investigar y desarrollar nuevas técnicas de control de estos sistemas para la mejora de la eficiencia.

Con el fin de realizar un estudio de técnicas de control para la mejora de la eficiencia, en este artículo se presenta un modelo para un caso realista de estudio donde se incorporan máquinas comerciales. Sin embargo, crear modelos que sean de interés desde un punto de vista práctico es una tarea difícil [1] debido, sobre todo, a la complejidad de la máquina enfriadora y a la falta de información detallada que ofrecen los fabricantes. Las empresas no disponen o bien no comparten modelos físicos precisos de las máquinas que producen.

Por este motivo, en este trabajo se propone la utilización de modelos basados en datos proporcionados por el fabricante o obtenidos mediante técnicas de identificación basadas en datos de ensayo. Dado que los fabricantes proporcionan información sobre índices de consumo y eficiencia energética, los modelos derivados permitirán definir índices de desempeño de tipo económico que incorporen esta información, siendo pues más realistas. Esto permitirá diseñar controladores óptimos desde el punto de vista económico.

Los diferentes elementos cuyos modelos se presentan son: válvula, intercambiador de calor, máquina enfriadora, y edificio. Concretamente, los modelos para intercambiador de calor y máquina enfriadora se han obtenido basados en datos de máquinas comerciales de un determinado fabricante.

Con el objetivo de probar el modelo desarrollado, se expondrá un caso de estudio de control predictivo basado en modelo [2], el cual se ha probado que puede llegar a ahorrar hasta un 30\% de energía en diversos sistemas, en comparación con técnicas de control más clásicas [8]. Esta técnica de control, aunque suele requerir mayor carga computacional 
respecto a otras técnicas, es muy estudiada en la actualidad por permitir optimizar un balance entre el coste energético y las condiciones de comfort, además de incorporar restricciones sobre variables críticas tales como la temperatura de congelación del fluido refrigerante.

La estructura de este artículo es la siguiente: la Sección 2 presenta una descripción general de un sistema de refrigeración usando agua como refrigerante, la Sección 3 describe el modelado de las unidades que se encuentran en el interior de las salas, la Sección 4 aborda el modelado de una máquina enfriadora, la Sección 5 considera el modelo del edificio y sus salas, la Sección 6 detalla la integración de los diferentes componentes del sistema, y por último la Sección 7 desarrolla un caso de control aplicado basado en modelo.

\section{DESCRIPCIÓN}

En este artículo se considera un sistema de refrigeración de planta de edificio utilizando agua como refrigerante con topología a caudal constante. El esquema se muestra en la Fig. 1 para un caso simple de dos salas.

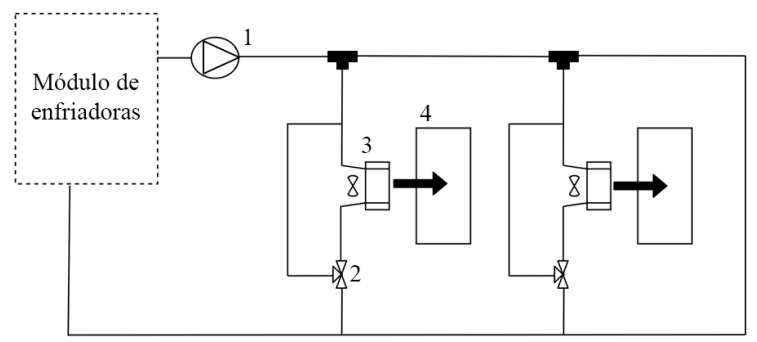

Figura 1: Esquema del sistema de refrigeración. Etiquetas: 1. Bomba de agua; 2. Válvula de tres vías; 3 . Intercambiador de calor; 4 . Salas.

El sistema se compone de un módulo de máquinas enfriadoras, la red de distribución de agua, el edificio, y múltiples unidades de interior, una para cada una de las salas del edificio. Las unidades de interior, a su vez, se componen de un intercambiador de calor y un ventilador.

El módulo de enfriadoras se encarga de refrigerar el caudal de agua procedente de la recirculación, proporcionando la potencia frigorífica del sistema. El caudal ya refrigerado es impulsado por una bomba hacia la red de tuberías, donde un conjunto de bifurcaciones ya prefijadas distribuyen un caudal parcial constante hacia las salas. En cada sala existe una unidad interior que intercambia calor, en este caso absorbiendo calor de la sala para refrigerar el aire interior. El ventilador de la unidad interior no es manipulable. La única forma de controlar el flujo de potencia que se produce en la unidad es a través de una válvula de tres vías que se sitúa a la salida de la sala. Esta válvula sí es controlable, y manipula el caudal parcial. Una parte pasará por el intercambiador y otra por un canal de derivación donde no se produce intercambio. Tras las válvulas controlables de cada sala, los caudales parciales se mezclan y se recirculan de nuevo al módulo de enfriadoras.

\section{MODELADO DE LAS UNIDADES DE INTERIOR}

En esta sección se presenta el modelado del conjunto compuesto por intercambiador de calor y válvula de tres vías controlable, que conforman la unidad de interior que refrigera cada sala. Por cada unidad, pasa un caudal parcial constante $q_{p}$ en litros por minuto.

Las válvulas son lineales y tienen un parámetro de apertura $A$ que comprende el rango $(0,1)$. Esta apertura $A$ divide el caudal parcial $q_{p}$ entre el que pasa por el intercambiador de calor de la sala $q_{p s}$ y el que se desvía por el canal de derivación $q_{p d}$ tal que

$$
\begin{aligned}
& q_{p s}=q_{p} * A \\
& q_{p d}=q_{p} *(1-A) .
\end{aligned}
$$

El intercambiador de calor, o fancoil en inglés, es un bobinado de tubos por donde circula el agua enfriada, y sobre los cuales un ventilador mueve el aire de la sala para intercambiar calor por convección forzada. El aire de la sala se enfría al ceder calor al agua que pasa por los tubos.

Para modelar el intercambiador de calor se ha optado por un modelo de regresión basado en datos, los cuales provienen de una hoja de datos de un dispositivo comercial. La potencia calorífica $P$ $(\mathrm{kW})$ que absorbe un intercambiador depende del caudal de agua que pasa por el intercambiador $q_{p s}(\mathrm{l} / \mathrm{min})$ y de la temperatura de entrada de este caudal $T_{p e}\left({ }^{\circ} \mathrm{C}\right)$. Para ajustar el modelo se ha utilizado la función polinomial no lineal de tercer grado

$$
\begin{aligned}
P= & p_{00}+p_{10} T_{p e}+p_{01} q_{p s}+p_{20} T_{p e}^{2}+ \\
& p_{11} T_{p e} q_{p s}+p_{02} q_{p s}^{2}+p_{30} T_{p e}^{3}+ \\
& p_{21} T_{p e}^{2} q_{p s}+p_{12} T_{p e} q_{p s}^{2}+p_{03} q_{p s}^{3} .
\end{aligned}
$$

Los valores de los coeficientes se muestran en la Tabla 1.

Figura 2 representa la ecuación 2. La potencia es negativa por convenio: el intercambiador produce frío extrayendo calor de la sala. Se puede observar que la potencia en módulo aumenta con el caudal y es inversamente proporcional a la temperatura. 
Tabla 1: Parámetros del intercambiador.

\begin{tabular}{|l|r|r|r|}
\hline$p_{x y}$ & Valor & $p_{x y}$ & \multicolumn{1}{|c|}{ Valor } \\
\hline$p_{00}$ & 467.3 & $p_{02}$ & 19.54 \\
\hline$p_{10}$ & -158.3 & $p_{30}$ & -0.4593 \\
\hline$p_{01}$ & -902.7 & $p_{21}$ & 0.0156 \\
\hline$p_{20}$ & 15.95 & $p_{12}$ & -0.3116 \\
\hline$p_{11}$ & 26.61 & $p_{03}$ & -0.139 \\
\hline
\end{tabular}

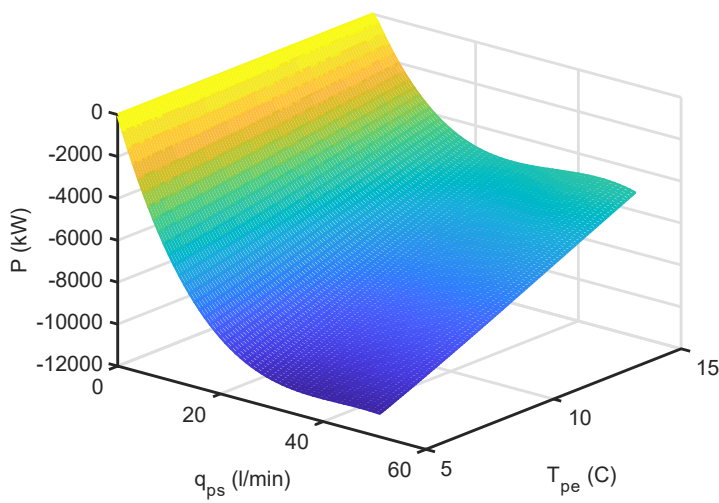

Figura 2: Modelo del intercambiador de calor. Valor de la potencia calorífica intercambiada en kilovatios en función de el caudal que pasa por el intercambiador en litros por minuto y la temperatura de entrada de este caudal en grados centígrados.

\section{MODELO ECONÓMICO DE ENFRIADORA}

En la actualidad, las máquinas refrigeradoras tienen un diseño complejo y varían según el fabricante. Además, sus planos y configuraciones internas son información confidencial de las empresas. Dada la dificultad de trabajar con un modelo físico preciso de una enfriadora real, se ha optado por obtener un modelo de parámetros económicos basado en datos para reproducir curvas de rendimiento [4], [9].

Los tres aspectos más importantes de nuestro modelo económico son la capacidad de la enfriadora, el factor de carga parcial, y el consumo eléctrico. La capacidad de una máquina enfriadora $(C A P)$ se define como la potencia frigorífica máxima que pueda aportar al fluido a refrigerar. El factor de carga de parcial $f c p$ es el porcentaje entre la potencia frigorífica real y la máxima $(C A P)$. Por último, el consumo eléctrico $C_{E}$ es la potencia que consume la máquina refrigeradora de la red eléctrica para poder funcionar. Las curvas que se presentarán de estos parámetros siguen una forma estandarizada en las herramientas de simulación de edificios de referencia [10].
La capacidad de una enfriadora $C A P$ no es un parámetro constante, sino que depende sobre todo de la temperatura del aire exterior $T_{a e}\left({ }^{\circ} \mathrm{C}\right)$ y de la temperatura de la salida del fluido a refrigerar $T_{w s}\left({ }^{\circ} \mathrm{C}\right)$. Se ha obtenido una función de esta capacidad

$$
C A P=C A P_{N} \cdot f\left(T_{a e}, T_{w s}\right)
$$

que depende de un parámetro constante denominado capacidad nominal $C A P_{N}$ propio de la máquina y de un factor modificador dado por la expresión

$$
\begin{aligned}
f\left(T_{a e}, T_{w s}\right)= & c_{0}+c_{1} T_{w s}+c_{2} T_{w s}^{2}+ \\
& c_{3} T_{a e}+c_{4} T_{a e}^{2}+c_{5} T_{a e} T_{w s}
\end{aligned}
$$

donde los coeficientes $c_{i}$ han sido obtenidos mediante identificación basada en datos de una hoja del fabricante. Los valores de dichos coeficientes se recogen en la Tabla 2.

Tabla 2: Parámetros del $C A P$ de la refrigeradora.

\begin{tabular}{|c|c|c|c|}
\hline$c_{x}$ & Valor & $c_{x}$ & Valor \\
\hline$c_{0}$ & 0.90491362 & $c_{3}$ & 0.00284939 \\
\hline$c_{1}$ & 0.04080004 & $c_{4}$ & -0.00016049 \\
\hline$c_{2}$ & 0.00052899 & $c_{5}$ & -0.00048742 \\
\hline
\end{tabular}

El factor de carga parcial se define como

$$
f c p=\frac{c_{p} q\left(T_{w e}-T_{w s}\right)}{C A P}
$$

donde $c_{p}$ es la capacidad calorífica del fluido a refrigerar en unidades del SI, $q$ es el caudal total de fluido en $\mathrm{l} / \mathrm{s}$, y $T_{w e}$ es la temperatura de entrada del fluido en ${ }^{\circ} \mathrm{C}$.

El consumo eléctrico $C_{E}$ también está basado en datos y sigue la siguiente función

$$
C_{E}=C_{E N} \cdot f^{\prime}\left(T_{a e}, T_{w s}\right) \cdot f^{\prime \prime}(f c p)
$$

que depende de un consumo nominal constante $C_{E N}$ asociado al dimensionamiento de la máquina, y de dos factores modificadores

$$
\begin{array}{r}
f^{\prime}\left(T_{a e}, T_{w s}\right)=q_{0}+q_{1} T_{w s}+q_{2} T_{w s}^{2}+ \\
q_{3} T_{a e}+q_{4} T_{a e}^{2}+q_{5} T_{a e} T_{w s} \\
f^{\prime \prime}(f c p)=k_{0}+k_{1} f c p+k_{2} f c p^{2}+k_{3} f c p^{3}
\end{array}
$$

donde nuevamente los coeficientes $q_{i}$ y $k_{i}$ se han obtenido por identificación a partir de datos por ensayo. Los valores de dichos coeficientes se recogen en la Tabla 3.

La Fig. 3 representa el polinomio 6 utilizando temperatura de aire exterior constante a $35{ }^{\circ} \mathrm{C}$. El consumo aumenta principalmente cuando lo hace 
Tabla 3: Parámetros del $C_{E}$ de la refrigeradora.

\begin{tabular}{|c|c|c|r|}
\hline$c_{x}$ & Valor & $c_{x}$ & \multicolumn{1}{|c|}{ Valor } \\
\hline$q_{0}$ & 35234 & $q_{5}$ & $7.6567 \times 10^{-5}$ \\
\hline$q_{1}$ & 60985 & $k_{0}$ & 0.20172656 \\
\hline$q_{2}$ & 52087 & $k_{1}$ & 0.13658742 \\
\hline$q_{3}$ & 32867 & $k_{2}$ & 0.68734829 \\
\hline$q_{4}$ & 15652 & $k_{3}$ & 0.02566227 \\
\hline
\end{tabular}

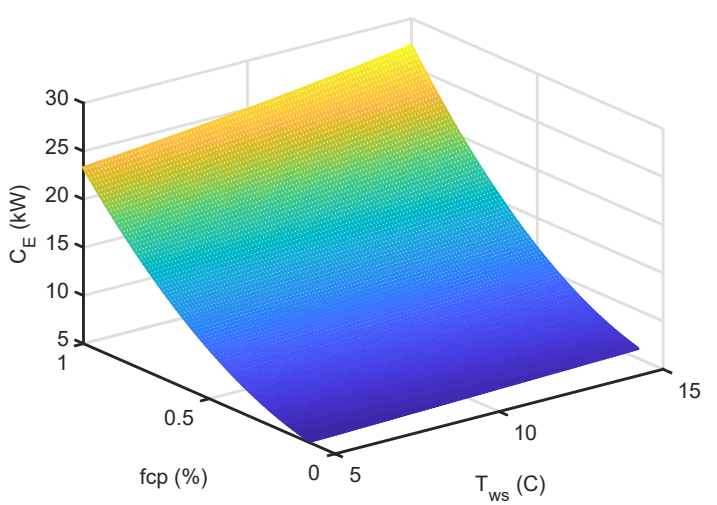

Figura 3: Modelo económico de la refrigeradora. Valor del consumo eléctrico de la máquina en kilovatios en función del factor de carga y de la temperatura del caudal de salida en grados centígrados.

el factor de carga parcial, ya que actúa directamente sobre la potencia frigorífica.

Una forma de medir la eficiencia energética consiste en calcular el coeficiente denominado $E E R$ [6], o coeficiente de eficiencia energética, que se define como el cociente entre la potencia frigorífica útil y el consumo eléctrico de la máquina. Este coeficiente permite encontrar puntos de funcionamiento de alta eficiencia energética, y se puede relacionar con los parámetros anteriormente descritos mediante la expresión

$$
E E R=\frac{f c p \cdot C A P}{C_{E}} .
$$

Por tanto, según las ecuaciones 3 y 6 , el $E E R$ depende de $T_{w s}, T_{a e}$, y $f c p$.

En la figura 4 se representa el coeficiente EER frente a $f c p$ y $T_{w s}$. Se puede observar que se obtienen valores óptimos de $E E R$ para valores de en torno a $f c p=0.5$.

\section{MODELADO DEL EDIFICIO}

En general, elaborar un modelo fidedigno de un edificio no es una tarea trivial. Existe una gran multitud de factores que se han de tener en cuenta, no solo relativos a la estructura y distribución

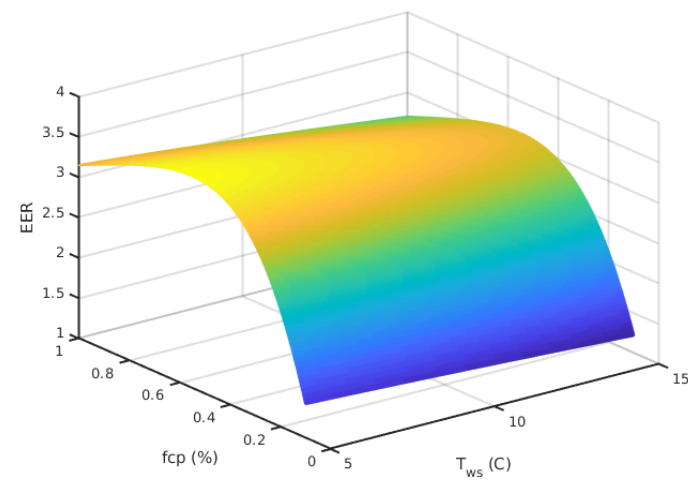

Figura 4: Coeficiente de eficiencia energética. Valor del EER en función del factor de carga y de la temperatura del caudal de salida en grados centígrados.

del espacio del edificio, sino también diversos factores que afectan a la dinámica de este. Ante este panorama, una opción es la obtención de un modelo por identificación en el que solo se tengan en cuenta los flujos de potencia y la temperatura de las salas. Sin embargo, este tipo de metodologías de modelado pueden hacer que diversas dinámicas asociadas a las no linealidades del sistema no se tengan en cuenta. Por esta razón, se ha optado por otra alternativa, que es la de utilizar un modelo complejo no lineal que tenga en cuenta el balance termodinámico de todos los elementos que intervienen.

Para el modelado aquí llevado a cabo, se ha utilizado la Toolbox BRCM para MATLAB [12]. Esta nos permite realizar modelos no lineales de edificios considerando no solo la estructura y distribución de las salas, sino también las cargas térmicas y los flujos de potencia. Dado que el interés principal de este trabajo no es el de explicar esta herramienta, solo se detallará el modelo obtenido del edificio y su integración con el resto de modelos de máquinas.

El modelo considerado del edificio para nuestro caso de estudio tiene tres salas, como puede observarse en la Fig. 5. El suelo y el techo de todas las salas se consideran adibáticos. En las paredes exteriores existe una transferencia de energía con el ambiente exterior por medio de convección y conducción. Las paredes internas también permiten el intercambio de calor entre salas adyacentes si existe un gradiente de temperatura entre ellas.

El modelo del edicifio recibe como entradas las cargas térmicas internas de las salas, temperatura del aire exterior, radiación solar, la temperatura inicial de las salas, y la potencia frigorífica que reciben. Como salida, el modelo devuelve la tem- 


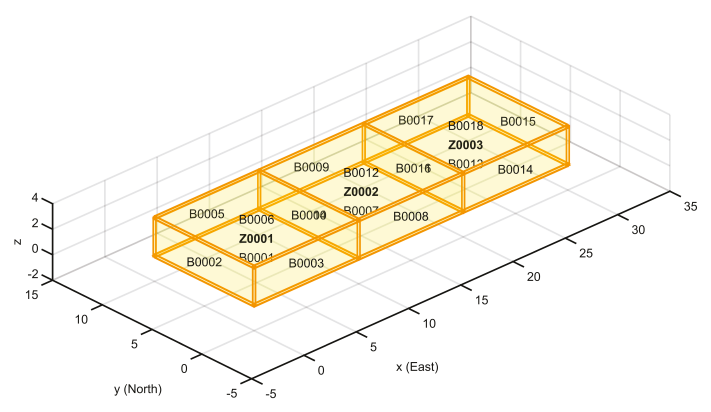

Figura 5: Estructura del edificio. Configuración y dimensionamiento en metros de las tres salas del modelo.

peratura de las salas transcurrido un tiempo de muestreo, que es un parámetro de cálculo interno configurable. Entre estas entradas, la temperatura inicial es una medición, la potencia frigorífica viene del modelo de intercambiador, y el resto de parámetros externos son estimaciones.

\section{INTEGRACIÓN DEL MODELO}

Una vez se tienen los modelos de válvula, intercambiador, edificio, y enfriadora, la jerarquía de conexión entre ellos sería la que se representa en la Fig. 6.

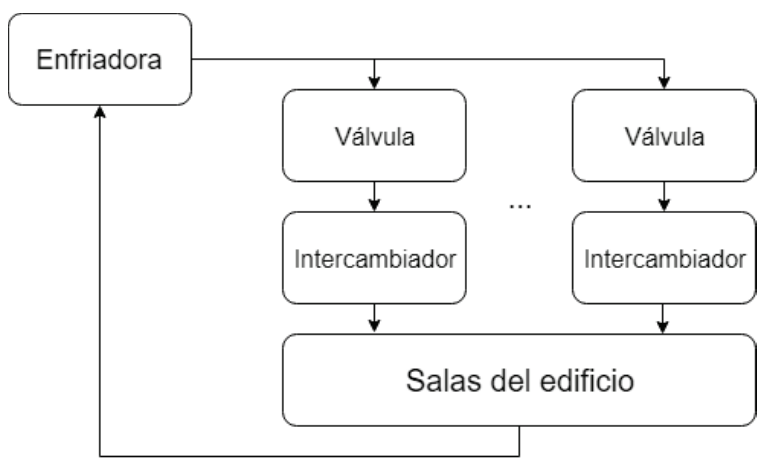

Figura 6: Diagrama de bloques del interconexionado entre los diferentes elementos modelados que componen el sistema.

Partiendo de los bloques de válvulas, las entradas son el caudal parcial de agua $q_{p}$, que es constante, y su temperatura $T_{p e}$, que es igual que la temperatura de salida de la enfriadora $T_{w s}$ en caso de despreciar las pérdidas. Las salidas de las válvulas son el caudal parcial a la sala $q_{p s}$. En el intercambiador, las entradas son $q_{p s}$ y $T_{p e}$, y la salida es la potencia de intercambio $P$. A partir de esta potencia, se puede calcular la temperatura de salida del agua del intercambiador de cada sala $T_{p s}$ como

$$
T_{p s}=\frac{P}{c_{p} q}+T_{p e} .
$$

La entrada a las salas del edificio es la potencia $P$ a cada sala, y la salida del bloque del edificio son las temperaturas de las salas. La entrada del bloque de enfriadora es la suma de caudales parciales, que se calcula como

$$
q=\sum_{i} q_{p}
$$

y la temperatura de entrada del agua a la enfriadora $T_{w e}$, que se deriva de un balance de energía y resulta ser la la media ponderada de las temperaturas de los caudales parciales

$$
T_{w e}=\frac{\sum_{i}\left(T_{p s} * q_{p s}+T_{p e} * q_{p d}\right)}{q} .
$$

La salida del bloque de enfriadora es la temperatura de salida del agua $T_{w s}$, completando el ciclo.

\section{CASO DE CONTROL APLICADO}

En este apartado, se muestra la aplicación de un controlador MPC [5] para seguimiento de referencias [3], [7] sobre el modelo no lineal ya presentado. En el modelo que vamos a utilizar existe una máquina enfriadora y tres salas de un edificio, cada una con su unidad de interior asociada.

El controlador diseñado se basa en un modelo linealizado del modelo no lineal, formulado en espacio de estados

$$
\begin{aligned}
x(k+1) & =A x(k)+B u(k) \\
y(k) & =C x(k),
\end{aligned}
$$

donde $x \in \mathbb{R}^{n x}$ con $n x=4$ es el estado del sistema, $u \in \mathbb{R}^{n u}$ con $n u=4$ es el conjunto de acciones de control, e $y \in \mathbb{R}^{n y}$ son las salidas medidas con $n y=4$. Las acciones de control son el factor de carga parcial $f c p$ de la enfriadora y las tres aperturas de válvula $A$ para cada una de las tres salas. Las salidas medidas son la temperatura de salida del agua de la enfriadora $T_{w s}$ y las tres temperaturas de las habitaciones $T_{r}$.

Para calcular el estado del sistema lineal a partir de las salidas de la planta no lineal, se ha diseñado un observador de estado. Como existen discrepancias entre ambos modelos, dicho observador tiene en cuenta perturbaciones de carácter aditivo en las salidas medidas del sistema

$$
\begin{aligned}
x(k+1) & =A x(k)+B u(k) \\
y(k) & =C x(k)+d(k),
\end{aligned}
$$

donde $d \in \mathbb{R}^{n y}$ son las perturbaciones sobre la salida medida. 
Para incluir las perturbaciones en el formato tradicional de espacio de estados, el estado del sistema se ha aumentado

$$
\mathbf{x}=\left[\begin{array}{l}
x \\
d
\end{array}\right]
$$

tal que

$$
\begin{aligned}
\mathbf{x}(k+1) & =\left[\begin{array}{cc}
A & 0 \\
0 & I
\end{array}\right] \mathbf{x}+\left[\begin{array}{c}
B \\
0
\end{array}\right] u \\
y(k) & =\left[\begin{array}{ll}
C & I
\end{array}\right] \mathbf{x} .
\end{aligned}
$$

A partir de este sistema aumentado, se resuelve un problema LQR para obtener la ganancia del observador $L$. Finalmente, el modelo del observador es el siguiente

$$
\begin{aligned}
\hat{\mathbf{x}}(k+1)= & \left(\left[\begin{array}{ll}
A & 0 \\
0 & I
\end{array}\right]-L\left[\begin{array}{ll}
C & I
\end{array}\right]\right) \hat{\mathbf{x}}(k) \\
& +\left(\left[\begin{array}{c}
B \\
0
\end{array}\right] \quad L\right) \mathbf{u},
\end{aligned}
$$

donde se ha utilizado la estructura clásica de un filtro de Kalman, siendo

$$
\mathbf{u}=\left[\begin{array}{l}
u \\
y
\end{array}\right] .
$$

En cada instante de muestreo, el observador estima el siguiente estado del sistema a partir de las entradas $u$ aplicadas y las salidas $y$ medidas. Este estado estimado es el estado inicial $\hat{x}_{0}$ que recibe el MPC.

El MPC resuelve el siguiente problema de optimización

$$
\begin{aligned}
\min _{x, u, x_{s}, u_{s}} & \sum_{k=0}^{N-1}\left[\begin{array}{l}
x(k)-x_{s} \\
u(k)-u_{s}
\end{array}\right]^{\prime}\left[\begin{array}{cc}
Q & 0 \\
0 & R
\end{array}\right]\left[\begin{array}{l}
x(k)-x_{s} \\
u(k)-u_{s}
\end{array}\right] \\
& +\left[x(N)-x_{s}\right]^{\prime} P\left[x(N)-x_{s}\right] \\
& +\left[y_{s}+d-y_{\text {ref }}\right]^{\prime} S\left[y_{s}+d-y_{\text {ref }}\right]
\end{aligned}
$$

$$
\begin{array}{ll}
\text { s.t. } & x(0)=\hat{x}_{0} \\
& x_{s}=A x_{s}+B u_{s} \\
& y_{s}=C x_{s} \\
& x(k+1)=A x(k)+B u(k) \\
& y(k)=C x(k) \\
& y \in \mathcal{Y} \\
& y_{s} \in \mathcal{Y} \\
& u \in \mathcal{U} \\
& u_{s} \in \mathcal{U}
\end{array}
$$

donde $x_{s} \in \mathbb{R}^{n x}$ es un estado estacionario, $u_{s} \in$ $\mathbb{R}^{n u}$ es la acción de control en el estado estacionario, $y_{s} \in \mathbb{R}^{n y}$ es la salida medida en el estado estacionario, $y_{\text {ref }} \in \mathbb{R}^{n y}$ es la referencia de la salida, $x_{0} \in \mathbb{R}^{n x}$ es el estado inicial, $N$ es el horizonte de predicción, $\mathcal{Y}$ es el conjunto de restricciones en la salida, y $\mathcal{U}$ es el conjunto de restricciones en las acciones de control.

La función de coste (19) junto a las restricciones de igualdad (19b) - (19e) se usan para establecer estabilidad y seguimiento de referencia. Las restricciones de desigualdad (19f) - (19i) imponen las restricciones en las entradas y salidas. Las matrices $Q>0$ y $R>0$ son los pesos de ponderación del coste de etapa. El coste terminal es dado por la matriz $P$, obtenida resolviendo la ecuación de Riccati

$$
A^{\prime} P A-P-\left(A^{\prime} P B\right)\left(R+B^{\prime} P B\right)\left(B^{\prime} P A\right)+Q=0 .
$$

La matriz $S$ pondera el coste de seguimiento, donde se han elegido valores diagonales tales que el seguimiento de las temperaturas de las habitaciones $T_{r}$ tengan un peso mucho mayor que la temperatura de salida de la refrigeradora $T_{w s}$, ya que es importante que el seguimiento de las temperaturas de las salas tenga erorres muy pequeños, mientras que la temperatura de salida de la refrigeradora solo debe cumplir que esté dentro de las restricciones.

Se ha realizado una simulación en bucle cerrado con el control MPC lineal conectado al sistema no lineal. El control MPC se conecta en el instante $t=200$ min para que el sistema se estabilice en el punto de funcionamiento y el estado estimado por el observador converja.

La Fig. 7 muestra las salidas del sistema durante la simulación. Se puede observar en las tres salas cómo la temperatura de cada una de ellas sigue su referencia (línea discontinua en negro). Además, la temperatura de salida del agua se mantiene estable dentro de sus restricciones, que son entre $1^{\circ} \mathrm{C}$ para evitar congelación y $15^{\circ} \mathrm{C}$ que asegura no salirse del rango de validez de los modelos.

Por otro lado, la Fig. 8 muestra las acciones de control del sistema durante la simulación. El factor de carga parcial $f c p$ aumenta si la demanda de frío es mayor entre las tres salas. Las aperturas de válvulas $A$ controlan cada sala individualmente, y son mayores cuando la potencia de frío requerida aumenta.

\section{CONCLUSIONES}

En este trabajo se ha presentado el modelo de un sistema de refrigeración orientado a la gestión de la eficiencia energética. Para ello, se han desarrollado modelos económicos basados en datos de máquinas comerciales enfriadoras e intercam- 

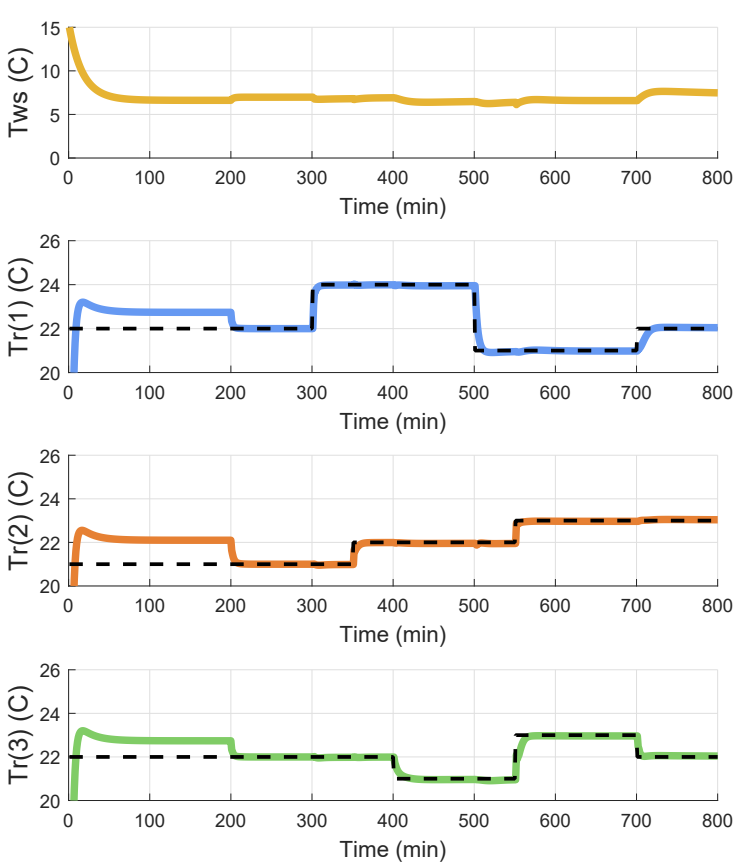

Figura 7: Salidas del sistema en la simulación en bucle cerrado con control MPC. Temperatura del caudal de salida de la enfriadora y temperatura de las salas del edificio.
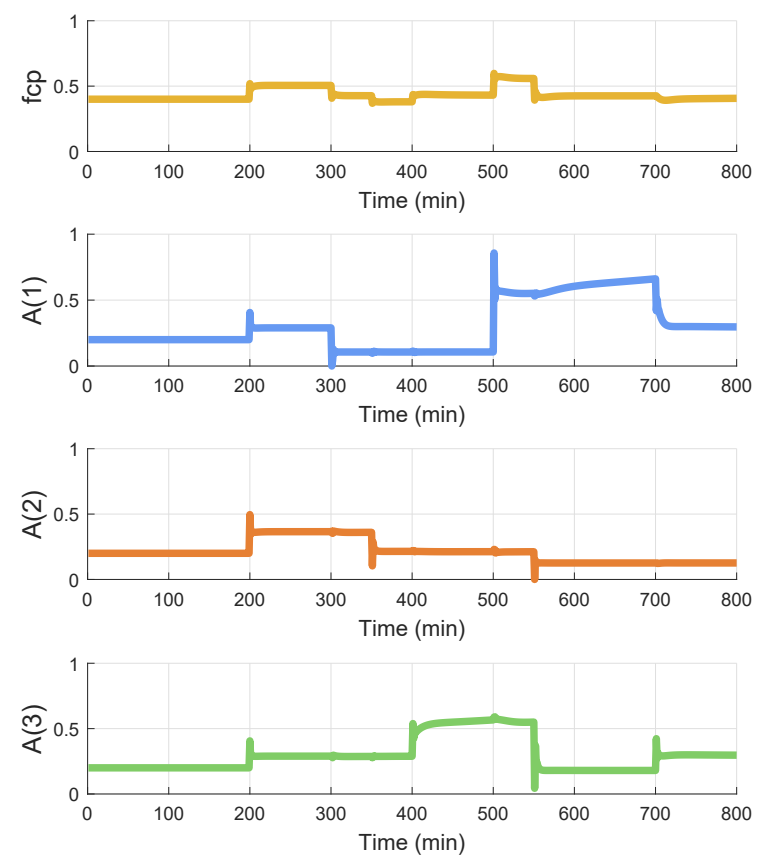

Figura 8: Entradas del sistema en la simulación en bucle cerrado con control MPC. Factor de carga (\%) y apertura de las válvulas de las distintas salas $(\%)$. biadores de calor, donde se tiene en cuenta el consumo y el rendimiento de ellas. Además, se ha desarrollado un caso de estudio de control utilizando MPC lineal para el seguimiento de temperaturas de referencia en las salas del edificio.

Como trabajo futuro, se propone explotar la modularidad de los diferentes modelos de máquinas para satisfacer la demanda energética de edificios de mayores dimensiones.

\section{Agradecimientos}

Los autores agradecen el apoyo y financiación del Ministerio de Economía y Empresa de España y fondos FEDER bajo el proyecto DPI2016-76493C3-1-R.

\section{English summary}

\section{MODELING OF REFRIGERA- TION SYSTEMS FOR CONTROL AND ENERGY EFFICIENCY}

\begin{abstract}
Building air conditioning is an important application of refrigeration systems, and its operation consumes up to $20 \%$ of the total energy in developed countries. Currently, control engineers are expend time and effort to find new methods that improve energy efficiency. However, obtaining proper models is a difficult task due to the complexity of comercial chillers and business confidentiality. In this paper, data-based identification is proposed to obtain non-linear models of real machines. Furthermore, a case study is presented with the application of model predictive control for temperature tracking.
\end{abstract}

Keywords: air conditioning, refrigeration, economic model, energy effciency, data-based identification, predictive control

\section{Referencias}

[1] Abdul Afram and Farrokh Janabi-Sharifi, Review of modeling methods for hvac systems, Applied Thermal Engineering 67 (2014), no. $1-2,507-519$. 
[2] Eduardo F Camacho and Carlos Bordons Alba, Model predictive control, Springer Science \& Business Media, 2013.

[3] Antonio Ferramosca, Daniel Limón, Ignacio Alvarado, Teodoro Alamo, and Eduardo F Camacho, Mpc for tracking with optimal closed-loop performance, 2008 47th IEEE Conference on Decision and Control, IEEE, 2008, pp. 4055-4060.

[4] Mark Hydeman and Kenneth L Gillespie, Tools and techniques to calibrate electric chiller component models, ASHRAE transactions 108 (2002), no. 1, 733-741.

[5] Roger Kwadzogah, Mengchu Zhou, and Sisi $\mathrm{Li}$, Model predictive control for hvac systems - a review, Automation Science and Engineering (CASE), 2013 IEEE International Conference on, IEEE, 2013, pp. 442-447.

[6] Harvey S Leff and William D Teeters, Eer, cop, and the second law efficiency for air conditioners, American Journal of Physics 46 (1978), no. 1, 19-22.

[7] Daniel Limón, Ignacio Alvarado, Teodoro Alamo, and Eduardo F Camacho, Mpc for tracking piecewise constant references for constrained linear systems, Automatica 44 (2008), no. 9, 2382-2387.

[8] Amin Mirakhorli and Bing Dong, Occupancy behavior based model predictive control for building indoor climate-a critical review, Energy and Buildings 129 (2016), 499-513.

[9] Danielle Monfet and Radu Zmeureanu, Identification of the electric chiller model for the energyplus program using monitored data in an existing cooling plant, Proceedings of the international IBPSA conference. Sidney, Australia: International Building Performance Simulation Association, 2011.

[10] US Department of Energy, Energyplus documentation, 2009.

[11] Luis Pérez-Lombard, José Ortiz, and Christine Pout, A review on buildings energy consumption information, Energy and buildings 40 (2008), no. 3, 394-398.

[12] David Sturzenegger, Dimitrios Gyalistras, Vito Semeraro, Manfred Morari, and Roy S Smith, Brcm matlab toolbox: Model generation for model predictive building control, 2014 american control conference, IEEE, 2014, pp. 1063-1069.

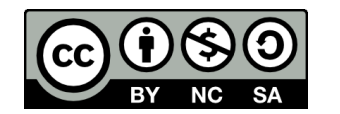

(c) 2019 by the authors. Submitted for possible open access publication under the terms and conditions of the Creative Commons Attribution CC BY-NC-SA 4.0 license (https://creativecommons.org/licenses/by-ncsa/4.0/deed.es). 